\title{
The relationship between anterograde and retrograde amnesia in alcoholic Wernicke-Korsakoff Syndrome ${ }^{1}$
}

The Wernicke-Korsakoff Syndrome (WKS) can be described as a constellation of neuropsychological deficits the most prominent of which is a profound amnesic syndrome. The neurological impairment underlying WKS is directly attributable to thiamine deficiency, which has the consequential effect of Wernicke's encephalopathy, resulting in vascular lesions of the midline diencephalic nuclei (Victor et al. 1989). The most common aetiology of this thiamine deficiency is chronic alcoholism, although it must be emphasized that the same form of neurological impairment can arise in other forms of nutritional disorder (e.g. Becker et al. 1990).

WKS patients exhibit both a severe anterograde amnesia, manifest by poor performance on memory tasks such as free recall and recognition, and retrograde amnesia, as shown by an inability to recall or recognize information relating to the pre-morbid period. Reviews of WKS indicate that the co-occurrence of anterograde and retrograde amnesia is a persistent feature of the syndrome (Parkin \& Leng, 1988). Indeed, to my knowledge, there is no well-described instance of WKS in which this relationship has not been reported. This regular state of affairs leads directly to the possibility that the anterograde and retrograde deficits may have a common origin.

The consistent association of WKS with chronic alcoholism has been invoked in attempts to provide a single explanation for the anterograde and retrograde deficits associated with WKS. This view, which has been termed the continuity hypothesis (e.g. Ryback, 1971), suggests that there is a continuum between the neurological impairments arising from chronic alcoholism (e.g. Parsons $e t$ al. 1987) and those sustained following an identifiable Wernicke's episode. Within this view the acute anterograde impairment observed at diagnosis can be considered as an exacerbation of a milder anterograde impairment present in the pre-morbid period. The apparent retrograde amnesia is therefore more properly considered the consequence of a milder anterograde impairment. As well as accounting for the regular co-occurrence of anterograde and retrograde impairments, the hypothesis also accounts for the characteristic temporal gradient in alcoholic WKS whereby performance on remote tests declines systematically as more recent portions of the pre-morbid period are sampled. The continuity view attributes this temporal gradient to the cumulative effects of chronic alcoholism in the pre-morbid period which leads to increasingly poorer registration of information.

The continuity view cannot easily be tested directly because data are rarely available about a WKS patient's remote memory prior to diagnosis (but, see below). However, one potential prediction of this view is that the severity of the anterograde and retrograde deficits might be expected to correlate if they are both, in fact, anterograde deficits. Several research workers have provided data relevant to this possibility. Shimamura \& Squire (1986) correlated the performance of Korsakoff patients on the Boston Remote Memory Test with their anterograde memory impairment as measured by 12 learning measures. Although substantial remote memory impairments were observed, no overall correlation between the extent of these impairments and anterograde amnesia was found. However, more detailed analyses did indicate a significant positive relationship between their anterograde deficit and retrograde amnesia for the most recent decade of the pre-morbid period. Furthermore these correlations remained significant when the data from the two most recent decades were pooled.

\footnotetext{
' Address for correspondence: Dr A. J. Parkin, Laboratory of Experimental Psychology, University of Sussex, Brighton, East Sussex BNI 9QG.
} 
More recently Kopelman (1989) has also examined the relationship between anterograde and retrograde amnesia in a group of Korsakoff patients. Anterograde amnesia was assessed in terms of a composite 'memory quotient' comprising subjects' scores on a range of recall and recognition tasks drawn from standardized memory tests. Retrograde amnesia was measured using four different tests, event memory, famous personalities, personal semantic memory, and autobiographical memory. Overall no significant correlations were found but, when only performance on the most recent period of remote memory was considered, correlations with the anterograde measures hovered around significance.

Research on our own group of alcoholic WKS patients (see Leng \& Parkin, 1989, for details) has revealed a picture similar to that of the above studies. We correlated performance of our patients on a prototype famous faces test for UK populations (see Parkin et al. 1990a) with performance on a variety of anterograde learning tests. These findings, summarized by Parkin (1990), indicated no overall correlation between the anterograde and retrograde measures but, when only retrograde performance in the most recent pre-morbid period was considered, significant correlations with anterograde measures did emerge. A further aspect of these data was that only anterograde tests measuring explicit, context dependent memory (e.g. Parkin et al. 1990 b), showed a significant correlation. Indirect, implicit, measures did not correlate significantly with retrograde amnesia.

The above data do not provide any strong basis for assuming a relationship between anterograde and retrograde deficits in alcoholic WKS. We note, however, that some correlation does exist when only the most recent section of the pre-morbid period is considered - a point I shall return to later. First we will consider other evidence pertinent to the issue.

It can be argued that the largely negative correlational data do not provide conclusive evidence against a common explanation for anterograde and retrograde amnesia in WKS. One objection is that there is no a priori basis on which to assume that the severity of a pre-morbid anterograde impairment should, of necessity, determine the additional impairment arising from the acute lesions associated with Wernicke's encephalopathy. Fortunately other lines of evidence do allow us to settle the issue.

Butters (1984) reports the case of P.Z., an academic scientist with a history of chronic heavy drinking which culminated in a full-blown WKS. Two years prior to this, P.Z. wrote his autobiography and this provided a hard index that the patient's remote memory prior to WKS was reasonably good. Post-morbidly P.Z.'s memory for facts in his autobiography was extremely impaired and showed a temporal gradient similar to that shown by alcoholic WKS patients on standardized remote memory tests. It seems difficult to accommodate this case within a continuity framework because this would predict preservation of remote memory at approximately the same level as it was pre-morbidly. Instead the data support an acute-onset interpretation of WKS retrograde amnesia.

A further important source of data comes from the few cases of WKS of non-alcoholic origin in which assessments of remote memory have been reported. Three apparent cases have so far been observed (Beatty et al. 1988; Becker et al. 1990; Parkin et al. 1990 c) and in all three the patients showed a pattern of anterograde impairment similar to alcoholic WKS and temporally graded retrograde amnesia. As none of these patients had a history of chronic alcoholism one can only conclude that their retrograde deficit had an acute onset and, on the grounds of parsimony, the same deficit in alcoholic WKS should also be interpreted as an acute phenomenon unrelated to premorbid alcoholism.

The available evidence therefore argues against chronic pre-morbid alcoholism as the factor linking anterograde and retrograde amnesia in WKS. An alternative possibility is that the two deficits have a common acute cause. An influential account of anterograde amnesia, including that in alcoholic WKS, is that patients have a selective impairment in the encoding of context (e.g. Mayes, 1988) - this term can be variously defined but one definition is that context is the information associated with a target trace that allows its discrimination from other, potentially similar, traces. Parkin et al. (1990a) examined the possibility that anterograde and retrograde amnesia in WKS might arise from damage to a single contextual processing system which was 
involved, both in the encoding of new contextual information, and the maintenance of existing contextual relationships in long-term storage. If true, this theory predicts that remote memory performance should be enhanced by contextual cueing in the same way that anterograde deficits in WKS can be ameliorated by contextual cues (Mayes, 1988). To test this idea the authors examined performance on a famous-faces test under two conditions - 'no context', in which minimal extraneous information concerning a personality's identity was provided, and a 'context' condition in which some obvious clue as to the person's identity was provided. Cueing was found to have progressively less of an effect as more recent periods of remote memory were sampled. A contextual deficit account of the alcoholic WKS retrograde amnesia impairment would have predicted the opposite and, as such, the data indicated that the impairment was more plausibly interpreted as a storage deficit. This interpretation is supported by Squire et al. (1989), who demonstrated that alcoholic WKS patients show no improvement when tested repeatedly on the same remote memory tests. This is consistent with a storage-deficit account but incompatible with a retrieval account because the latter would, on the assumption that the accessibility of memories would vary across time, predict a cumulative increase in overall performance.

The one finding we are left with is the observation that correlations have been found between anterograde deficits and remote memory tests restricted to the most recent section of the pre-morbid period. One interpretation of this relates to the difficulty in specifying, in the case of many patients, the exact point at which WKS commenced. WKS can often have an insidious onset (Cutting, 1978) and it is quite possible that significant correlations between anterograde amnesia and tests addressing only the most recent pre-morbid period are a reflection of an evolving anterograde impairment more characteristic of WKS than any milder deficit that may have been experienced at earlier stages of the pre-morbid period. However, even this relation between anterograde and retrograde amnesia might be questioned. Cohen \& Squire (1981) have argued that retrieval of remote memories might be partly determined by learning that occurs after the events themselves have occurred - the argument being that events continue to be discussed after their occurrence and that this contributes partly to their eventual memorability. If one assumes that recent remote memories are more likely to be strengthened by this process, then the correlation between anterograde amnesia and only recent remote memory can be attributed to a purely post-morbid memory impairment.

To sum up, we have found no evidence to suggest that anterograde and retrograde amnesia in alcoholic WKS reflect a common functional deficit. Instead the evidence points towards a storage deficit account of retrograde amnesia and the distinct possibility that anterograde amnesia involves some form of contextual encoding deficit. One should also note that anterograde and retrograde amnesia in WKS most likely involves damage to different structures in the mid-line diencephalon. This follows from case reports such as our own case M.G. (Hunkin \& Parkin, 1990), in which a hypothalamic glioma produced an anterograde impairment as severe as our alcoholic WKS patients but no remote memory impairment (for other examples of diencephalic amnesia without significant retrograde amnesia see Cohen $\&$ Squire, 1981; Winocur et al. 1984). This suggests, potentially, that a functional double dissociation might be found whereby a patient with a diencephalic pathology presents remote memory impairment in the absence of an anterograde impairment. Studies of temporal lobe amnesia have provided instances of this dissociation (Stuss \& Guzman, 1988) but, to my knowledge, it has not been shown in patients with diencephalic amnesia. Finally we should note that, as yet, there is no explanation of the temporal gradient in WKS retrograde amnesia. Parkin et al. (1990a) have suggested tentatively that the deficit might reflect the consequences of damage to a distributed memory system in which older memories are represented with a higher degree of redundancy which, in turn, makes those memories less vulnerable to brain lesions which partially destruct the system. Future research will address this possibility.

A. J. PARKIN 


\section{REFERENCES}

Beatty, W. W., Bailly, R. C. \& Fisher, L. (1988). Korsakoff-like amnesic syndrome in a patient with anorexia and vomiting. International Journal of Clinical Neuropsychology 11, 55-65.

Becker, J. T., Furman, J. M. R., Panisset, M. \& Smith, C. (1990). Characteristics of the memory loss of a patient with Wernicke Korsakoffs Syndrome without alcoholism. Neuropsychologia 28 , 171180.

Butters, N. (1984). Alcoholic Korsakoff's syndrome: an update. Seminars in Neurology 4, 226244.

Cohen, N. J. \& Squire, L. R. (1981). Retrograde amnesia and remote memory impairment. Neuropsychologia 19, 337-356.

Cutting, J. (1978). The relationship between Korsakov's syndrome and alcoholic dementia. British Journal of Psychiatry 132, 240251.

Hunkin, N. M. \& Parkin, A. J. (1990). Amnesic syndrome following hypothalamic glioma. (In preparation.)

Kopelman, M. D. (1989). Remote and autobiographical memory, temporal context memory and frontal atrophy in Korsakoff and Alzheimer patients. Neuropsychologia 27, 437460

Mayes, A. R. (1988) Human Organic Memory Disorders. Cambridge University Press: Cambridge.

Leng, N. R. C. \& Parkin. A. J. (1989). Aetiological variation in the amnesic syndrome: comparisons using the Brown-Peterson task. Cortex 25, 251.259.

Parkin, A. J. (1990). Recent advances in the neuropsychology of memory. In Clinical and Biochemical Contributions to the Understumeling of Human Memory Function (ed. J. Weinmann and J. Hunter). Harwood: London.

Parkin. A. J. \& Leng, N. R. C. (1988). Comparative studies of the amnesic syndrome. In IIformation Processing by the Brain: Views: and Hypotheses from a Phisiological-Cognitive Perspective (ed. $\mathrm{H}$. Markowitsch), pp. 107 123. Hans Huber: Toronto.

Parkin, A. J. Montaldi, D., Leng, N. R. C. \& Hunkin, N. M. $(1990 a)$. Contextual cueing effects in the remote memory of alcoholic Korsakoff patients. Quarler/y Journal of Experimental Psychology (in the press).

Parkin, A. J., Leng, N. R. C. \& Hunkin, N. M. (1990h). Differential sensitivity to contextual information in diencephalic and temporal lobe amnesia. Cortex (in the press).

Parkin, A. J. Blunden, J., Rees, J. \& Hunkin, N. M. (1990c). Wernicke-Korsakoff Syndrome of non-alcoholic origin. Brain and Cognition (in the press).

Parsons, O. A., Butters, N. \& Nathan, P. E. (1987). Neuropsychology of Alcoholism: Implications for Diagnosis and Treatment. Guilford: New York.

Ryback, R. (1971). The continuum and specificity of the effects of alcohol. Quarterly Journal of Studies in Alcoholism 32, 236242.

Shimamura, A P. \& Squire, L. R. (1986). Korsakoffs syndrome: the relationship between anterograde amnesia and remote memory impairment. Behavioral Neuroscience' 100, 165170.

Squire, L. R., Haist, F. \& Shimamura, A. P. (1989). The neurology of memory: Quantitative assessment of retrograde amnesia in two groups of amnesic patients. Journal of Neuroscience 9, 828839.

Stuss, D. T. \& Guzman, D. A. (1988). Severe remole memory loss with minimal anterograde amnesia. Brain and Cognition 8, 2130.

Victor, M., Adams, R. D. \& Collins, G. H. (1989). The Wernick' Korsakoff Syndrome, 2nd edn. Blackwells: Oxford.

Winocur, G., Oxbury, S., Roberts, R., Agnetti, V. \& Davis, C. (1984). Amnesia in a patient with bitateral lesions to the thalamus. Neuropsychologia 22, 123143. 